\title{
Probing the involvement of the earliest levels of cortical processing in motion extrapolation with rapid forms of visual motion priming and adaptation
}

\author{
Luca Battaglini • Gianluca Campana • \\ Rebecca Camilleri • Clara Casco
}

Published online: 12 November 2014

(C) The Psychonomic Society, Inc. 2014

\begin{abstract}
In this study, we investigated the effect of brief motion priming and adaptation, occurring at the earliest levels of the cortical visual stream, on time-to-contact (TTC) estimation of a target passing behind an occluder. By using different exposure times of directional motion presented in the occluder area prior to the target's disappearance behind it, our aim was to modulate (prime or adapt) the extrapolated motion of the invisible target, thus producing different TTC estimates. Our results showed that longer (yet subsecond) exposures to motion in the same direction as the target produced late TTC estimates, whereas shorter exposures produced shorter TTC estimates, indicating that rapid forms of motion adaptation and motion priming affect extrapolated motion. Our findings suggest that motion extrapolation might occur at the earliest levels of cortical processing of motion, at which these rapid mechanisms of priming and adaptation take place.
\end{abstract}

Keywords Motion extrapolation - Motion adaptation · Visual motion priming $\cdot$ Time to contact $\cdot \mathrm{TTC} \cdot \mathrm{rMAE} \cdot \mathrm{rVMP}$

In everyday life, moving objects frequently appear and disappear behind occluders, such as in the case of a motorbike passing beyond stationary cars and buses parked in front of us. Despite the disappearance of the motorbike, we are very good at estimating the time of the bike's reappearance between one vehicle and the other. This process has been

Luca Battaglini and Gianluca Campana contributed equally to this work

L. Battaglini · G. Campana $(\bowtie) \cdot$ R. Camilleri $\cdot$ C. Casco

Department of General Psychology, University of Padova,

Via Venezia 8, 35131 Padova, Italy

e-mail: gianluca.campana@unipd.it

G. Campana

Human Inspired Technology Research Centre (HIT),

University of Padova, Padova, Italy psychophysically studied using the prediction-of-motion (PM) paradigm, in which participants estimate the time to contact (TTC): Having seen the initial part of an object's trajectory prior to occlusion, they are asked to estimate when the object moving behind the occluder would reach a given point, usually the end of the occluder. By means of manipulating the variables related to the object's motion (e.g., velocity, occlusion distance, and/or duration) or other features (size, contrast, or shape), many studies have investigated the underlying processes involved in PM (e.g., Battaglini, Campana, \& Casco, 2013; Caird \& Hancock, 1994; DeLucia, 2004; Gray \& Regan, 2000; Schiff \& Oldak, 1990; Sokolov \& Pavlova, 2003).

Although it has been suggested that TTC is inferred by a "cognitive clocking" strategy (DeLucia \& Liddell, 1998; Tresilian, 1995), most studies have shown evidence that TTC is estimated via motion extrapolation, or imagined motion. This is when the object's trajectory, even when not visible behind the occluder, is tracked on the basis of memory of the object's motion before entering the occluder (de Sperati \& Deubel, 2006; Khoei, Masson, \& Perrinet, 2013; Makin \& Bertamini, 2014; Makin \& Poliakoff, 2011; Makin, Poliakoff, Ackerley, \& El-Deredy, 2012; Makin, Poliakoff, Chen, \& Stewart, 2008). In support of the view of visuo-perceptual processing underlying TTC estimation, it has been shown that it is affected by illusory changes in the speed of the moving object (Battaglini et al., 2013). Furthermore, it elicits a sequence of saccadic eye movements that follow the moving object even when it is occluded (Jonikaitis, Deubel, \& de Sperati, 2009), and it is affected by moving distractors (Lyon \& Waag, 1995), texture motion of the moving object (Oberfeld, Hecht, \& Landwehr, 2011), and visual motion adaptation (Gilden, Blake, \& Hurst, 1995). In the latter study, participants had to make a TTC judgment when a moving object disappeared beyond an occluder. However, before each trial, the region corresponding to the occluder was adapted 
with translational motion. This adaptation lasted $150 \mathrm{~s}$ on the first trial, and was followed by top-ups of $10 \mathrm{~s}$ on each successive trial. The results showed that when the target object moved in the same direction as the adaptation, TTC estimates were longer, whereas when the target object moved in the opposite direction with respect to that of adaptation, TTC estimates were shorter. The interpretation given by the authors was that adaptation of the occluder area biases the responses of motion detectors (with receptive fields sensitive to that area) toward the direction of motion opposite that of adaptation (similar to what occurs in the classical motion aftereffect [MAE]). This increases the motion signal of the imagined or extrapolated motion of the target object when its direction is opposite with respect to the adapted one, and decreases the motion signal of the imagined motion of the target object when its direction is the same with respect to the adapted one. The influence that prior adaptation had on TTC estimations suggests that the underlying mechanism is perceptual and engages the same neural structures involved in both motion imagery and motion perception-that is, intermediate-level extrastriate areas ranging from V2 to V5/ MT, to the parietal cortex (Goebel, Khorram-Sefat, Muckli, Hacker, \& Singer, 1998; Kaas, Weigelt, Roebroeck, Kohler, \& Muckli, 2010). The question that we posed in this study was the following: Which are the earliest cortical stages involved in TTC estimation and motion extrapolation?

In order to answer this question, we took advantage of recently investigated phenomena involving rapid forms of motion adaptation. Indeed, even exposures to directional stimuli much shorter than those used to elicit the classical MAE can bias the perceived motion direction of a subsequently presented ambiguous test pattern. Kanai and Verstraten (2005) showed that adaptation durations and interstimulus intervals around $120 \mathrm{~ms}$ are able to produce a bias in perception of a subsequent flickering stimulus in the same direction as the adaptation (rapid visual motion priming [rVMP]), whereas increasing the adaptation duration up to 320 $640 \mathrm{~ms}$ had the opposite effect, producing a rapid form of MAE (rMAE; Kanai \& Verstraten, 2005). Converging evidence from various psychophysical studies has suggested that these rapid forms of motion adaptation occur at an early stage of motion processing. For example, adaptation to a counterphase flickering pattern (ambiguous motion) has been used to probe the level of processing of motion adaptation. Despite simultaneously activating early motion detectors in opposite directions, ambiguous motion is usually perceived as directional, which is determined at higher levels of cortical processing (Williams, Elfar, Eskandar, Toth, \& Assad, 2003). Adaptation to ambiguous motion produces neither rVMP nor rMAE, suggesting that these two effects rely upon low-level sites of motion processing (Kanai \& Verstraten, 2005). In fact, ambiguous motion did produce a facilitation effect similar to that of priming, but with a slower time course than rVMP, suggesting that "a different kind of plasticity exists at a later stage" (Kanai \& Verstraten, 2005). Given the relative independence of first- and second-order motion at early stages of processing, transfer of adaptation (adapting with one and testing with the other) from first- to second-order motion (or vice versa) could also be used to investigate the level of processing of rapid forms of adaptation. Corroborating the low-level-processing hypothesis arising from the findings with ambiguous motion, it has been found that transfer between the two types of motion was small or absent: rVMP and rMAE can be elicited by both first- and second-order motion, but only if both the adapting and test stimuli are of the same motion type (first- or second-order; Pavan, Campana, Guerreschi, Manassi, \& Casco, 2009). Finally, by using components of the optic flow (complex motion) typically processed at intermediate and high levels of motion processing (Morrone et al., 2000; Wall, Lingnau, Ashida, \& Smith, 2008) to investigate the level of processing of rapid forms of adaptation, it was shown that, whereas rMAE can be elicited by both simple translational motion and complex motion, rVMP can only be produced with simple translational motion (Pavan, Campana, Maniglia, \& Casco, 2010). This finding proposes the notion that the faster the adaptation (and interstimulus interval; rVMP), the earlier the level of processing. All of these findings suggest an early locus of processing of these rapid effects, and this is particularly true for rVMP, which cannot be elicited by complex motion.

Neuro-interference studies have confirmed the idea that rMAE can be processed at intermediate and low levels of processing: Indeed, both the classical MAE and rMAE are more strongly reduced when either areas $\mathrm{V} 2 / \mathrm{V} 3$ or $\mathrm{V} 5 / \mathrm{MT}$ are disrupted with transcranial magnetic stimulation (TMS) during the interstimulus interval (Campana, Maniglia, \& Pavan, 2013; Campana, Pavan, Maniglia, \& Casco, 2011; Théoret, Kobayashi, Ganis, Di Capua, \& Pascual-Leone, 2002). With regard to motion priming, TMS studies implicate area V5/MT (but not area V1) in this form of implicit memory (Campana, Cowey, \& Walsh, 2002, 2006), whereas at present no neurointerference studies are available that have investigated the neural circuitries involved in the generation of rVMP.

The rationale behind this study is that, if motion extrapolation of the (invisible) moving object is indeed biased by rapid forms of adaptation of the same sorts that produce rMAE and rVMP, and since such adaptation occurs at early levels of visual motion processing, then motion extrapolation must also rely on these early levels of processing. The present study is in some ways similar to that of Gilden and colleagues (1995). The difference between these studies is that the present one goes a step further, by using not just the classical timing for generating the MAE, but implementing brief subsecond adaptation that might tap even earlier stages of motion processing, thereby producing either priming or aftereffect. 
Our results will show that both brief and longer (but still subsecond) adaptation durations are able to influence motion extrapolation, but with opposite results: When the direction of the adapting pattern was the same as that of the target (congruent), longer adaptation durations $(600 \mathrm{~ms})$ on the occluder area produced an increase in response times (late TTC estimation), whereas briefer adaptation durations (80 ms) on the occluder area produced a decrease in response times (early TTC estimation). These data suggest that the adaptation mechanisms that produce rMAE and rVMP, respectively, are able to influence motion extrapolation, and thus that early levels of visual processing are involved in motion extrapolation.

\section{Experiment 1}

\section{Method}

Participants Seventeen participants (ten female, seven male) including one of the authors (L.B.) participated in this experiment (they were between 21 and 35 years of age). All participants except the author were naive to the purpose of the experiment, and participants gave written informed consent according to the Declaration of Helsinki prior to their inclusion in the experiment. All participants had normal or corrected-to-normal visual acuity.

Apparatus Participants were seated in a dark room $57 \mathrm{~cm}$ from the display screen. Viewing was binocular; stimuli were generated with MATLAB and the Psychophysics Toolbox (Brainard, 1997; Pelli, 1997) and were displayed on a 19-in. CTX CRT Trinitron monitor with a refresh rate of $100 \mathrm{~Hz}$. The screen resolution was $1,024 \times 768$ pixels. Each pixel subtended $\sim 1.9$ arcmin. The maximum luminance was $125 \mathrm{~cd} / \mathrm{m}^{2}$, and the minimum luminance was $0.9 \mathrm{~cd} / \mathrm{m}^{2}$. Luminance was measured using a Minolta LS-100 photometer.

Stimuli The target stimulus was a small circle $(0.5 \mathrm{deg}$ in diameter, $125 \mathrm{~cd} / \mathrm{m}^{2}$ ), appearing $13 \mathrm{deg}$ to the left or the right from the center of the screen with equal probabilities (in order to avoid the buildup of directional aftereffects), and travelling with a horizontal trajectory toward the opposite side of the screen at a constant speed of either 3.02 or $6.05 \mathrm{deg} / \mathrm{s}$. The extent of the linear visible motion trajectory was $6.3 \mathrm{deg}$. After that, the target disappeared under an invisible occluder (same luminance as the background), which was $7.5 \mathrm{deg}$ in length and $2 \mathrm{deg}$ in height. A gray bar $(0.3 \mathrm{deg}$ in width $\times 4 \mathrm{deg}$ in height, $24 \mathrm{~cd} / \mathrm{m}^{2}$ ) represented the end of the invisible trajectory, whereas a red dot $\left(0.1\right.$ in diameter, $\left.24 \mathrm{~cd} / \mathrm{m}^{2}\right)$ placed $0.2 \mathrm{deg}$ above the center of the invisible tunnel was the fixation spot. Figure 1 shows a schematic representation of the stimuli.
An adapting random-pixel array texture was employed before the disappearance of the target. The texture had the same size and position as the invisible occluder (with no overlap with the gray bar) and was made by assigning a random value between 0 and 255 RGB to each pixel, with a mean luminance of $28 \mathrm{~cd} / \mathrm{m}^{2}$. The texture could have three types of motion: (i) in the same direction as the target's motion (congruent condition), (ii)in the opposite direction with respect to the target's motion (incongruent condition), and (iii)no net motion direction (random noise, control condition). The speed of the texture with a specific direction was $16 \mathrm{deg} / \mathrm{s}$. A high speed was chosen in order to avoid any kind of plausible cue of speed (or time) that could allow inference of the TTC of the target, whose motion was much slower, and also in order to increase the strength of the aftereffect on the (imagined) motion of a target with lower speed (Bex, Verstraten, \& Mareschal, 1996; Campana et al., 2013). In the randomnoise condition, each pixel changed its RGB value randomly every $40 \mathrm{~ms}$, producing the effect of a detuned TV.

The adapting texture could last either $600 \mathrm{~ms}$ (long adaptation) or $80 \mathrm{~ms}$ (brief adaptation). These are the stimulus durations capable of producing, respectively, rMAE and rVMP (Kanai \& Verstraten, 2005; Pavan et al., 2009; Pavan et al., 2010). The texture was always removed $120 \mathrm{~ms}$ before the target reached the end of the visible trajectory (before disappearing behind the occluder). As soon as the texture disappeared, the visible gray bar was displayed.

Experimental procedure Participants were instructed to maintain fixation on the central red spot and to press the spacebar when they thought the leading edge of the moving target (imagining that it maintained the same constant speed and direction behind the occluder) reached a gray bar indicating the end of the invisible trajectory (TTC estimation). The intertrial interval was $1 \mathrm{~s}$, and no performance feedback was given. Moreover, participants were instructed to ignore a texture patch, which was briefly displayed before the disappearance of the target. The two texture durations (long adaptation [600 ms] vs. brief adaptation [80 ms]) were displayed separately in two different blocks. Each block consisted of 20 repetitions for each combination of speed (low speed [3\% $/ \mathrm{s}$. high speed $[6 \%$ ] $)$ by texture motion (congruent, incongruent, or random noise), for a total of 120 trials, randomly presented.

\section{Results}

We analyzed the mean TTC of the invisible trajectory estimates with a three-way repeated measures analysis of variance (ANOVA), with Adaptation Duration (long vs. brief), Speed (low vs. high), and Texture Motion (congruent, incongruent, random noise) as the factors. Since for Texture Motion the sphericity of the data was violated, as indicated by a significant Mauchly's test $\left(W_{2}=0.58, p<.05\right)$, the Greenhouse- 


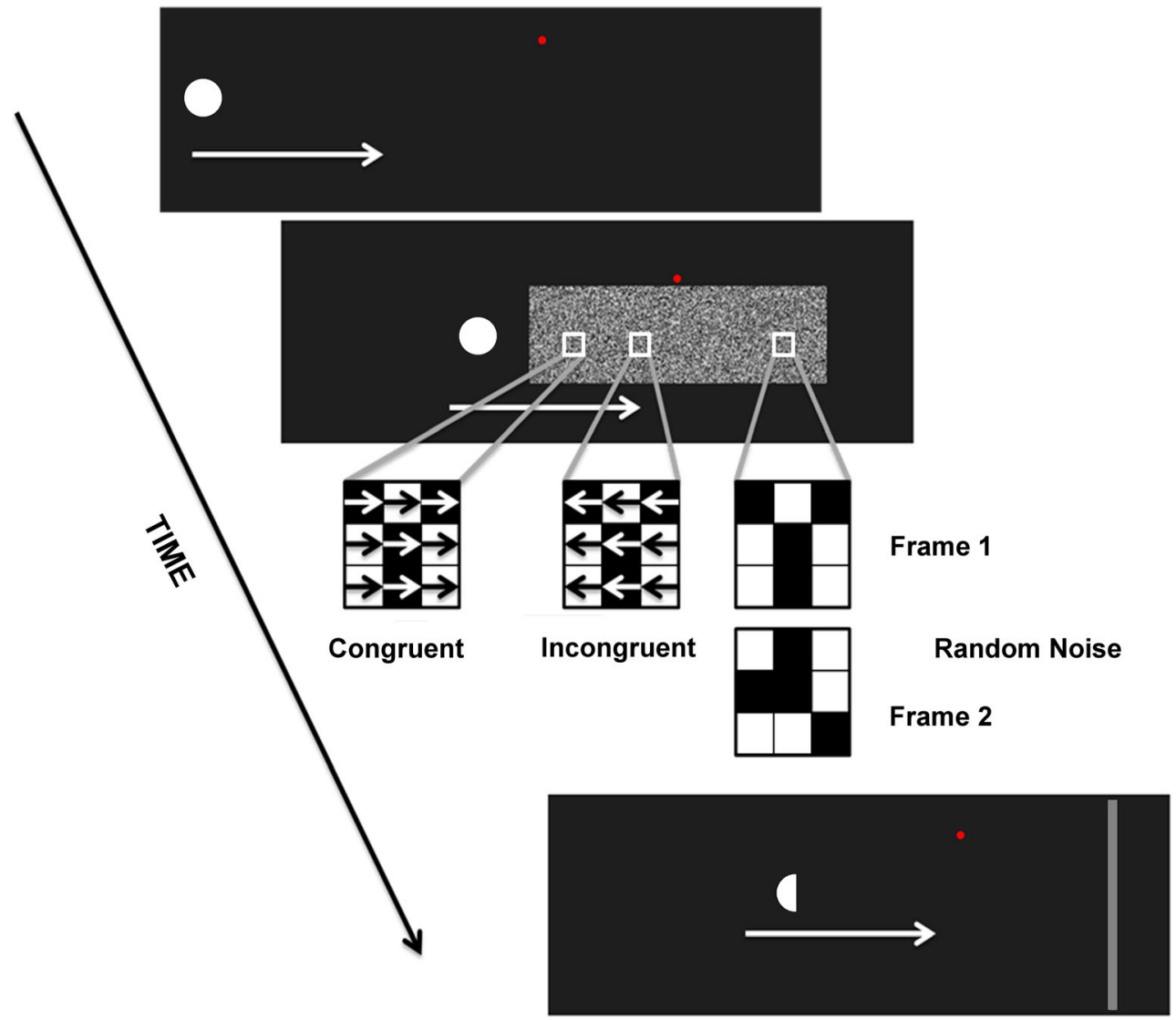

Fig. 1 Illustration of a trial in Experiment 1. A moving target traveled along a linear path at a constant speed. Then, a texture appeared for either $600 \mathrm{~ms}$ (long adaptation) or $80 \mathrm{~ms}$ (short adaptation). The texture could move congruently (same direction with respect to the target's motion) or incongruently (opposite direction with respect to the target's motion), or it could contain dynamic random noise (no directional energy). The texture

Geisser correction for the degrees of freedom was used for this factor.

The ANOVA showed a significant effect of speed $[F(1$, 16) $\left.=792.16, p<.0001, \eta_{\mathrm{p}}{ }^{2}=.98\right]$ and a significant interaction of texture motion with adaptation duration $\left[F(2,32)=8.21, p<.005, \eta_{\mathrm{p}}^{2}=.34\right]$.

The effect of speed was as expected, since faster targets indeed produced lower TTC estimates.

Bonferroni-corrected post-hoc $t$ tests indicated that, for both long and brief adaptations (able to produce, respectively, rMAE and rVMP), a significant difference (of opposite signs for the two adaptation durations) was apparent between congruent and incongruent texture motion. In other words, long adaptation produced longer $[t(16)=-3.92, p<.005]$ TTC estimates for congruent motion $(2.1 \mathrm{~s})$ with respect to the incongruent motion condition ( $2.02 \mathrm{~s})$. With long adaptation, we also found significantly longer TTC estimates $[t(16)=$ $-3.17, p<.05]$ for congruent motion $(2.1 \mathrm{~s})$ with respect to random noise ( $2.03 \mathrm{~s})$ (Fig. 2). These results suggest that the imagined motion of the target was slowed when the occluder area was adapted in the same direction as the target, with was removed $120 \mathrm{~ms}$ before the end of the visible trajectory of the target. Finally, the participants had to press a button when they thought that the moving target (imagining that it maintained the same constant speed and direction behind the occluder) reached the gray bar, indicating the end of the invisible trajectory

respect to the condition in which the occluder area was adapted in the opposite motion direction (or even with random noise) from that of the target. Overall, these results suggest that the same mechanism implied in rMAE is able to modulate imagined motion of the target behind an occluder.

On the other hand, brief adaptation produced shorter $[t(16=3.57, p<.01]$ TTC estimates for congruent motion $(1.98 \mathrm{~s})$ with respect to the incongruent motion condition (2.06 s; Fig. 3), paralleling the results obtained with rVMP (Kanai \& Verstraten, 2005).

The absence of differences between either the congruent or the incongruent condition and random noise (with the exception of a significant difference between congruent motion and random noise with long adaptation and high speed) may be due to the small size of the effect $(\sim 100 \mathrm{~ms})$ on a judgment on the order of seconds, in conjunction with the high variability between participants (there was a twofold difference in TTC estimates between the fastest and slowest participants), yielding a significant betweensubjects effect $\left[F(1,16)=688, p<.0001, \eta_{\mathrm{p}}{ }^{2}=.34\right]$, reflected in the large error bars reported in the figures. 


\section{Long Adaptation}

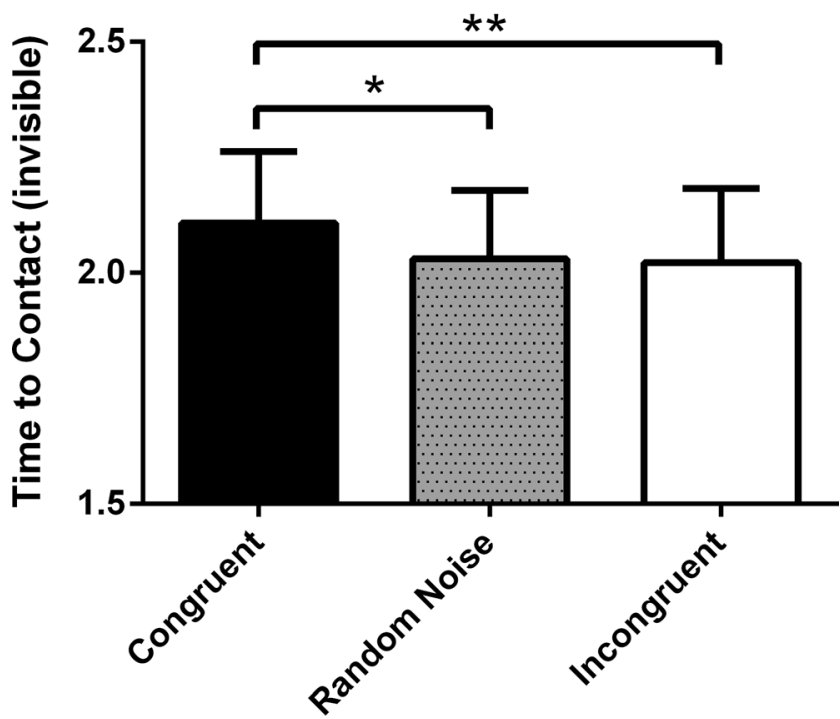

Texture Motion

Fig. 2 Long adaptation: Results of the block with a long-duration adapting texture $(n=17)$. Motion adaptation $(600 \mathrm{~ms})$ with a congruent moving texture (black column) produced late response times (TTC estimation) relative to incongruent (white column) and random-noise (gray column) textures. Error bars indicate 1 SEM. ${ }^{*} p<.05 .{ }^{* *} p<.01$

This is probably due to the fact that the TTC estimation task is a subjective judgment, and thus highly variable.

It is still possible, though, that the present results reflect changes in the perceived speed of the visible target, rather than in its imagined speed once occluded. Indeed, we have recently shown (Battaglini et al., 2013) that TTC judgments are highly dependent on the perceived speed of the target stimulus prior to occlusion. However, since here the presentation of the adapting stimulus occurred during the late portions of the target motion trajectory, this hypothesis is quite unlikely. However, in order to test this, we ran a control experiment in which we measured the perceived target speeds with the different experimental configurations (short- vs. long-duration adapting texture, congruent vs. incongruent direction of the adapting texture with respect to the target motion).

\section{Experiment 2}

To test whether the adapting texture could modify the perceived speed of the visible target before occlusion, we measured the point of subjective equality (PSE) with a twointerval forced choice using two simple one up-one down staircases interleaved in the same block. Stimuli from the descending and ascending staircase conditions were presented randomly within the same block.

\section{Brief Adaptation}

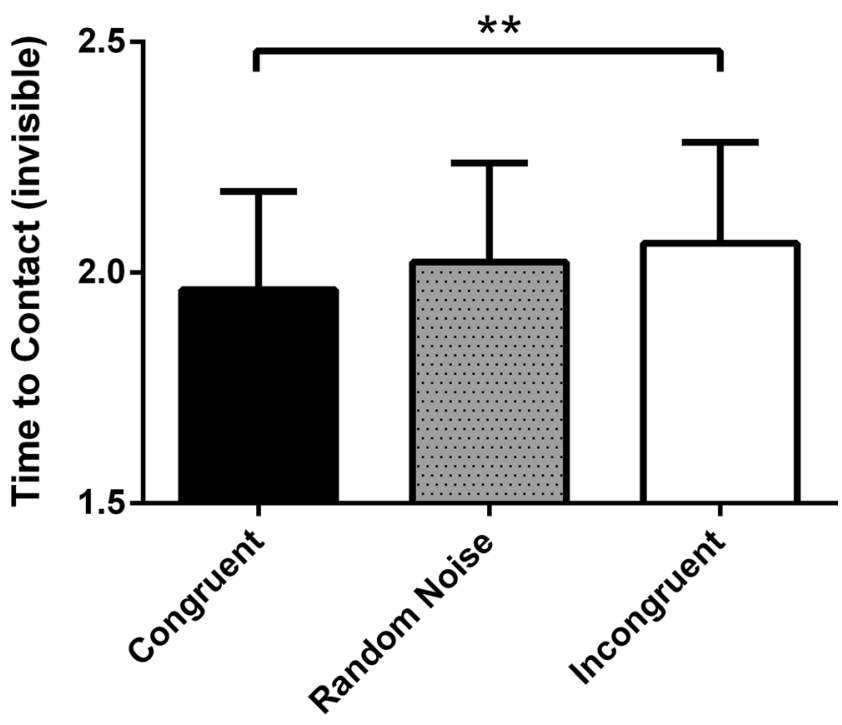

\section{Texture Motion}

Fig. 3 Brief adaptation: Results of the block with a short-duration adapting texture $(n=17)$. Motion adaptation $(80 \mathrm{~ms})$ with a congruent moving texture (black column) sped up response times (TTC estimation) relative to the incongruent moving texture (white column). Error bars indicate $1 S E M .{ }^{* *} p<.01$

\section{Method}

Participants Eighteen students from the University of Padova (nine female, nine male; ages 19-22 years) took part voluntarily in this experiment. The participants had normal or corrected-to-normal vision and were required to give written informed consent according to the Declaration of Helsinki.

Stimuli and apparatus The apparatus was the same as in the previous experiment, as well as the target and texture features. Two different moving targets with the same behavior were presented in two different temporal windows. The target that kept the same speed throughout the experiment ( 3.02 or $6.05^{\circ} \%$ s) was referred to as a standard stimulus (SS), whereas the comparison stimulus (CS) was the target that changed its speed according the participant's response. The starting position, direction, and end of the visible trajectory were the same as in Experiment 1. After $1 \mathrm{~s}$ from the disappearance of the target, the first temporal window ended, and the second moving target appeared. The congruent moving texture was always presented with the SS, whereas the incongruent texture was always presented with the CS. We contrasted the congruent and incongruent moving textures instead of using a random-noise texture as the SS because our aim was to maximize the possibility of finding possible biases due to the texture motion direction. The onset and duration of the texture 


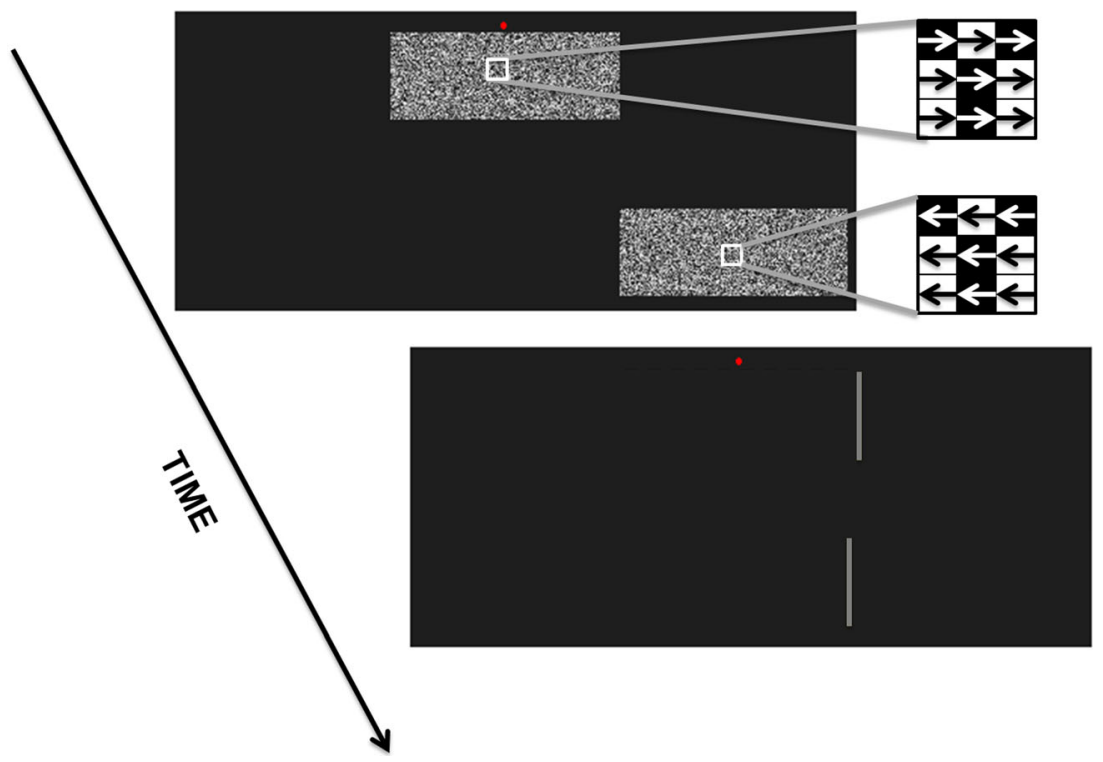

Fig. 4 Illustration of a trial in Experiment 3. Two moving textures appeared for either $600 \mathrm{~ms}$ (long adaptation) or $80 \mathrm{~ms}$ (short adaptation). One texture was presented just below the fixation point, and the other texture could be presented either on the right (like the one shown here) or on the left. The two textures could move inward, as illustrated above, or

were exactly the same as in the previous experiment according to the different block conditions (brief vs. long adaptation).

Experimental procedure Participants were asked to keep their fixation on the central red dot and to indicate by pressing one of the two response buttons (vertically aligned) which of the two intervals contained the faster target. The next trial then started $1.5 \mathrm{~s}$ after the participant's response. Participants performed four blocks: (1)SS with low speed $(3.02 \%$ s) and brief adaptation $(80 \mathrm{~ms}),(2) \mathrm{SS}$ with high speed $\left(6.05^{\circ} \mathrm{s}\right)$ and brief adaptation (80 ms), (3) SS with low speed $(3.02 \%$ s) and long adaptation (600 ms), and (4) SS with high speed (6.05\%) and long adaptation $(600 \mathrm{~ms})$. The starting speed of the CS was half of and double the SS speed for the ascending and descending staircases, respectively. The initial step of the staircase was set at $10 \%$ of the SS speed. After each reversal, the step size was halved until $0.1 \%$ of the SS speed was attained. The adaptive procedure could stop either after a total of 64 trials or after 12 reversals, whichever came first. The mean speed of the CS across the last eight reversals of the ascending and descending staircases was averaged and taken as a PSE threshold estimate (Campana et al., 2011; McKee, Klein, \& Teller, 1985).

\section{Results}

We compared the speed of the SS with the PSE of the CS resulting from each of the four blocks/experimental conditions with a one-sample $t$ test. The results indicated that, in none of the conditions was the perceived speed of the CS (target with outward. The textures were removed $120 \mathrm{~ms}$ before the onset of the Vernier stimulus, which lasted for $100 \mathrm{~ms}$. Finally, the participants had to indicate whether the upper line of the Vernier stimulus was displaced to the left or the right of the lower line

the incongruent moving texture) different from the speed (either slow, $3.02 \%$ s, or fast, $6.05 \%$ ) of the SS (target with the congruent moving texture): slow speed, brief adaptation: $3.06 \%$ s $[t(16)=1.3, p>.05]$; slow speed, long adaptation: $3.01 \%$ s $[t(16)=0.23, p>.05]$; high speed, brief adaptation: $6.12 \%$ s $[t(16)=0.71, p>.05]$; high speed, long adaptation: $6.2 \% \mathrm{~s}[t(16)=1.5, p>.05]$. This result rules out the possibility that the effects found in Experiment 1 were due to a modification of the perceived speed of the visible target trajectory by the moving texture presented just before target's disappearance.

\section{Experiment 3}

Another plausible possibility is that the effect of rapid adaptation on motion extrapolation could have been caused by a change in the perceived position of the invisible start of the occluder (the end of the occluder was marked by a gray bar, and may thus have been less susceptible to apparent position shifts). In fact, besides causing the MAE, motion adaptation is also able to produce a shift of position in the direction opposite the adapted one (position aftereffect [PA]; McGraw, Whitaker, Skillen, \& Chung, 2002), independently from the MAE (Whitney \& Cavanagh, 2003), and even when motion was just "implied" from static pictures (Pavan, Cuturi, Maniglia, Casco, \& Campana, 2011). Although PA has only been found with prolonged adaptations and with test stimuli overlapping the adapted region (McGraw et al., 2002; Pavan et al., 2011; 
Pavan \& Mather, 2008), it is possible that it can also be induced by brief adaptations and with test stimuli located at the edge of the adapted region. If so, this could also explain the results of our first experiment: After adaptation to the same direction as the target motion, the PA might increase the apparent width of the occluder by extending its invisible edge back in the direction of the PA, leading to longer TTC estimates. The opposite could occur for adaptation in the opposite direction to the target (shortening the occluder and therefore TTC). To test this hypothesis, we used a modified PA procedure with adapting textures and timing similar to Experiment 1, coupled with a Vernier task. Two vertically displaced adapting textures moving in opposite directions were displayed, and the lines of the Vernier task were subsequently presented at the edges of the adapted regions. Note that, by using two adapting textures and a Vernier, we increased (in fact, doubled) any PA, if present, with respect to the amount that could have been in Experiment 1, in which only one moving texture was presented.

\section{Method}

Participants Seventeen students from the University of Padova (nine female, eight male; ages 19-29 years) took part voluntarily in this experiment. The participants had normal or corrected-to-normal vision and were required to give written informed consent according to the Declaration of Helsinki.

Stimuli The apparatus was the same as in the previous experiments. Two adapting random-pixel array textures with the same characteristics as the previous experiments were employed and displayed before the appearance of the thin lines target (3.8-arcmin width) used to measure Vernier acuity. One of the textures had the same size and position as in the previous experiment, with respect to the fixation spot. The second texture had the same size but was placed 3 deg under the first and was displaced to either the left or the right of the first texture, so that an edge of the first texture was aligned with an edge of the second texture: When the second texture (lower in position) was presented on the left, its right edge was aligned with the left edge of the upper texture; when the second texture was presented on the right, its left edge was aligned with the right edge of the upper texture (Fig. 4). As in the previous experiments, the adapting texture could last either $600 \mathrm{~ms}$ (long adaptation) or $80 \mathrm{~ms}$ (brief adaptation). The Vernier stimulus (luminance $=24 \mathrm{~cd} / \mathrm{m}^{2}$ ) was always displayed $120 \mathrm{~ms}$ after the disappearance of the two textures and lasted for $100 \mathrm{~ms}$.

Experimental procedure Participants were instructed to maintain fixation on the central red spot, and the task of the experiment was, in all blocks, to indicate the position of the upper as compared to the lower line by means of a buttonpress. The two textures always moved in opposite directions: that is, inward or outward direction. Participants performed four blocks: (1)inward motion with brief texture durations ( $80 \mathrm{~ms}$ : brief adaptation condition); (2)outward motion with brief texture durations; (3)inward motion with long texture durations (600 ms: long adaptation condition); and (4) outward motion with long texture durations. The lower texture was presented at the left or the right part of the visual field with equal probabilities within each block. A trial started with the presentation of the brief or long adaptation texture, and after $120 \mathrm{~ms}$ a Vernier stimulus appeared at the same location as the aligned edges of the two textures, with a starting spatial offset along the $y$-axis of $2 \mathrm{deg}$. Within each block, four simple staircases (one up-one down; Levitt, 1971), one for each position of the lower texture (left and right) and one for each starting offset displacement (left and right), were used to calculate the PSE for perceiving the two Vernier lines as aligned. The initial step of each staircase was set at 0.2 deg. After each reversal, the step size was halved until it reached 1.9 arcmin (one pixel). Each staircase could stop either after a total of 64 trials or after 12 reversals, whichever came first.

If rapid adaptation, the effect of adaptation on the motion extrapolation found in Experiment 1, was caused by a change in the perceived position of the invisible start of the occluder, then we should also find a PA (apparent displacement of the two Vernier lines produced by the adapting textures) modulated by the type of motion (inward vs. outward) and by the adaptation duration (brief vs. long). In particular, we should find different PSEs (of opposite signs) with brief and long adaptations within each type of motion direction (inward or outward), and different PSEs (of opposite signs) with inward and outward motion within each adaptation duration (brief or long).

\section{Results}

We analyzed the mean PSEs of the Vernier lines with a twoway repeated measures ANOVA, with Adaptation Duration (long vs. brief) and Texture Motion Direction (inward vs. outward) as factors. The ANOVA did not show any significant effects of adaptation duration $[F(1,16)=3.17, p>.5$, $\left.\eta_{\mathrm{p}}{ }^{2}=.16\right]$, nor of motion direction $[F(1,16)=0.65, p>.5$, $\left.\eta_{\mathrm{p}}{ }^{2}=.04\right]$, nor of their interaction $[F(1,16)=0.002, p>.5$, $\left.\eta_{\mathrm{p}}{ }^{2}=.0001\right]$. Paired $t$ tests on the inward versus outward moving textures, performed separately for the brief [position shift $=$ 0.3 arcmin; $t(16)=-0.53, p>.05$ ] and long [position shift $=$ 0.3 arcmin; $t(16)=-0.46, p>.05$ ] adaptations, and on brief versus long adaptations, performed separately for the inward [position shift $=1.1 \operatorname{arcmin} ; t(16)=1.2, p>.05$ ] and outward [position shift $=1.1$ arcmin; $t(16)=1.9, p>.05$ ] moving textures, confirmed the null result of the interaction found with 
the ANOVA. In fact, the magnitude of the position shift found here was much smaller than that found with the classical PA (6 to 7 arcmin; McGraw et al., 2002), or than that found with implied motion with static photographs $(\sim 3$ arcmin; Pavan et al., 2011). It appears similar to the magnitude of the position shifts obtained with second-order motion (0.9 arcmin; Pavan \& Mather, 2008), although here the effect was nonsignificant, despite the fact that we used a larger sample of participants $(N$ $=17)$ than did Pavan and Mather $(N=7)$. We are aware that with a null result we cannot conclude that no PA takes place with rapid adaptation, but even if it did, its extent was so minuscule (the high-speed target can cover 1 arcmin in less than $3 \mathrm{~ms}$, a tiny fraction of the $\sim 100$-ms difference found in Exp. 1) that it could not influence the TTC estimates in our first experiment.

\section{General discussion}

We have shown that even a very brief exposure to directional motion is able to modulate TTC estimation. When the occluder area was adapted to congruent (same direction as the target's motion) directional motion just before the target disappeared behind it, longer (although subsecond) adaptation produced later TTC estimates with respect to adaptation to incongruent motion (opposite direction with respect to the target's motion). Briefer adaptation to congruent motion, on the other hand, produced earlier TTC estimates. These results demonstrate that motion extrapolation, on which TTC estimates are based, depends upon the activity of the same lowlevel motion detectors that are responsible for rMAE and rVMP. In fact, longer adaptation produces an imbalance between detectors tuned to opposite motion directions, lowering the activity of the adapted detectors and favoring the activity of nonadapted detectors, thus signaling the opposite motion direction with respect to the adapted one and producing rMAE. If the target's motion is congruent with the motion adaptation, the extrapolated motion of the target passing behind the adapted occluder will be hampered, due to lower activity of the detectors tuned to that motion direction.

On the other hand, the mechanism underlying briefer adaptation may consist of temporal integration between the adapting and the subsequent test stimulus (Pinkus \& Pantle, 1997), so that, if the test stimulus does not have a net motion direction, the motion direction signaled by the motion detectors will be that of the adapting stimulus, thus producing rVMP. In our experiments, if the target's motion was congruent to the motion prime (briefer adaptation), the extrapolated motion of the target passing behind the adapted occluder was facilitated.

The exact mechanism by which adaptation might produce an increasing or a lowering of the motion signal, thus increasing or decreasing the TTC, is still a matter of debate. It has been shown that prolonged adaptation modulates the perceived speed of a visible moving pattern (Hietanen, Crowder, \& Ibbotson, 2008). Although an effect of brief adaptation on perceived (or extrapolated) speed has never been demonstrated, we can speculate that the mechanism underlying the changes in TTCs due to brief adaptation resides in a modulation of the extrapolated speed of the target: Motion detectors responding to the target would sum the speed of the extrapolated motion with (or subtract from it) the speed resulting from brief adaptation.

It had already been shown that classical (long) motion adaptation of the occluder area could shift response times by producing late TTC estimates with adaptation in the same direction of the target, or by producing early TTC estimates with adaptation in the opposite direction from the target (Gilden et al., 1995). Those results imply that (a)TTC is performed via imagined or extrapolated motion, and (b)extrapolated motion relies on the activity of those neurons whose responsiveness is altered by adaptation, thus pointing to shared neural processes between extrapolated and real motion, located between areas V1 and MST (Gilden et al., 1995). In fact, numerous studies have already established the involvement of sensory and perceptual visual cortical areas in both mental imagery and working memory functions (see, e.g., Borst, Ganis, Thompson, \& Kosslyn, 2012; Kosslyn et al., 1999), on which motion extrapolation is likely to be based.

The primary aim of this study was to challenge the earliest levels of visual motion processing, in order to investigate whether or not they are involved in TTC estimation. In order to do so, we exploited perceptual effects arising from rapid exposure to motion, which are able to briefly modulate the responsiveness of motion detectors either in the direction opposite the adapted one (rMAE) or in the same direction (rVMP), depending on the adaptation time and interstimulus interval. Indeed, it has been shown that these rapid effects cannot be generated by high-level adaptation to counterphase flickering (Kanai \& Verstraten, 2005), and show little or no transfer between different types of motion that at low levels are processed independently (Pavan et al., 2009). Moreover, rMAE has been shown to depend upon the functional integrity of intermediate and low-level areas (Campana et al., 2011), and psychophysical experiments have shown that rVMP cannot be elicited by types of complex motion (Pavan et al., 2010) that are processed at intermediate and high levels of processing (i.e., from area $\mathrm{V} 3 \mathrm{a}$, thus excluding the involvement of the earlier areas V1 and V2; Wall et al., 2008). Finally, rapid forms of adaptation have been found to depend on both cortical and thalamic short-term depression (Carandini, Heeger, \& Senn, 2002; Chance, Nelson, \& Abbott, 1998; Chung, Li, \& Nelson, 2002).

So, although rMAE can occur at both low and intermediate levels of processing (Campana et al., 2011), making the level 
at which $600 \mathrm{~ms}$ of adaptation modulates TTC estimation questionable, rVMP has been shown to display characteristics compatible only with the earliest levels of processing (Pavan et al., 2010); therefore, an effect of very brief adaptation (the same that causes rVMP) on TTC estimation clearly points to the earliest levels of motion processing.

The results of the present study demonstrate that rapid forms of motion adaptation and motion priming affect motion extrapolation, suggesting that the mechanism underlying motion extrapolation can occur at even earlier levels of processing than those postulated by Gilden in 1995, possibly beginning at the level of primary visual cortex (V1).

\section{References}

Battaglini, L., Campana, G., \& Casco, C. (2013). Illusory speed is retained in memory during invisible motion. $i$-Perception, 4, 180 191.

Bex, P. J., Verstraten, F. A., \& Mareschal, I. (1996). Temporal and spatial frequency tuning of the flicker motion aftereffect. Vision Research, $36,2721-2727$.

Borst, G., Ganis, G., Thompson, W. L., \& Kosslyn, S. M. (2012). Representations in mental imagery and working memory: Evidence from different types of visual masks. Memory \& Cognition, 40, 204-217.

Brainard, D. H. (1997). The Psychophysics Toolbox. Spatial Vision, 10, 433-436. doi:10.1163/156856897X00357

Caird, J. K., \& Hancock, P. A. (1994). The perception of arrival time for different oncoming vehicles at an intersection. Ecological Psychology, 6, 83-109.

Campana, G., Cowey, A., \& Walsh, V. (2002). Priming of motion direction and area V5/MT: A test of perceptual memory. Cerebral Cortex, 12, 663-669.

Campana, G., Cowey, A., \& Walsh, V. (2006). Visual area V5/MT remembers "what" but not "where.". Cerebral Cortex, 16, 17661770. doi:10.1093/cercor/bhj111

Campana, G., Maniglia, M., \& Pavan, A. (2013). Common (and multiple) neural substrates for static and dynamic motion after-effects: A rTMS investigation. Cortex, 49, 2590-2594. doi:10.1016/j.cortex. 2013.07.001

Campana, G., Pavan, A., Maniglia, M., \& Casco, C. (2011). The fastest (and simplest), the earliest: The locus of processing of rapid forms of motion aftereffect. Neuropsychologia, 49, 2929-2934.

Carandini, M., Heeger, D. J., \& Senn, W. (2002). A synaptic explanation of suppression in visual cortex. Journal of Neuroscience, 22, 10053-10065.

Chance, F. S., Nelson, S. B., \& Abbott, L. F. (1998). Synaptic depression and the temporal response characteristics of V1 cells. Journal of Neuroscience, 18, 4785-4799.

Chung, S., Li, X., \& Nelson, S. B. (2002). Short-term depression at thalamocortical synapses contributes to rapid adaptation of cortical sensory responses in vivo. Neuron, 34, 437-446.

DeLucia, P. R., \& Liddell, G. W. (1998). Cognitive motion extrapolation and cognitive clocking in prediction motion tasks. Journal of Experimental Psychology: Human Perception and Performance, 24, 901-914. doi:10.1037/0096-1523.24.3.901

DeLucia, P. R. (2004). Time-to-contact judgments of an approaching object that is partially concealed by an occluder. Journal of
Experimental Psychology: Human Perception and Performance, 30, 287-304. doi:10.1037/0096-1523.30.2.287

de Sperati, C., \& Deubel, H. (2006). Mental extrapolation of motion modulates responsiveness to visual stimuli. Vision Research, 46, 2593-2601.

Gilden, D., Blake, R., \& Hurst, G. (1995). Neural adaptation of imaginary visual motion. Cognitive Psychology, 28, 1-16.

Goebel, R., Khorram-Sefat, D., Muckli, L., Hacker, H., \& Singer, W. (1998). The constructive nature of vision: Direct evidence from functional magnetic resonance imaging studies of apparent motion and motion imagery. European Journal of Neuroscience, 10, 1563 1573.

Gray, R., \& Regan, D. (2000). Risky driving behavior: A consequence of motion adaptation for visually guided motor action. Journal of Experimental Psychology: Human Perception and Performance, 26, 1721-1732. doi:10.1037/0096-1523.26.6.1721

Hietanen, M. A., Crowder, N. A., \& Ibbotson, M. R. (2008). Differential changes in human perception of speed due to motion adaptation. Journal of Vision, 8(11), 6.1-10.

Jonikaitis, D., Deubel, H., \& de Sperati, C. (2009). Time gaps in mental imagery introduced by competing saccadic tasks. Vision Research, 49, 2164-2175. doi:10.1016/j.visres.2009.05.021

Kaas, A., Weigelt, S., Roebroeck, A., Kohler, A., \& Muckli, L. (2010). Imagery of a moving object: The role of occipital cortex and human MT/V5+. NeuroImage, 49, 794-804. doi:10.1016/j.neuroimage. 2009.07.055

Kanai, R., \& Verstraten, F. A. (2005). Perceptual manifestations of fast neural plasticity: Motion priming, rapid motion aftereffect and perceptual sensitization. Vision Research, 45, 3109-3116.

Khoei, M. A., Masson, G. S., \& Perrinet, L. U. (2013). Motion-based prediction explains the role of tracking in motion extrapolation. Journal of Physiology, 107, 409-420.

Kosslyn, S. M., Pascual-Leone, A., Felician, O., Camposano, S., Keenan, J. P., Thompson, W. L., \& Alpert, N. M. (1999). The role of area 17 in visual imagery: Convergent evidence from PET and rTMS. Science, 284, 167-170.

Levitt, H. (1971). Transformed up-down methods in psychoacoustics. Journal of the Acoustical Society of America, 49(2, Pt.2), 467-477. doi:10.1121/1.1912375

Lyon, D. R., \& Waag, W. L. (1995). Time course of visual extrapolation accuracy. Acta Psychologica, 89, 239-260.

Makin, A. D., \& Bertamini, M. (2014). Do different types of dynamic extrapolation rely on the same mechanism? Journal of Experimental Psychology: Human Perception and Performance, 40, 1566-1579.

Makin, A. D., \& Poliakoff, E. (2011). Do common systems control eye movements and motion extrapolation? Quarterly Journal of Experimental Psychology, 64, 1327-1343.

Makin, A. D., Poliakoff, E., Ackerley, R., \& El-Deredy, W. (2012). Covert tracking: A combined ERP and fixational eye movement study. PloS One, 7, e38479. doi:10.1371/ journal.pone.0038479

Makin, A. D., Poliakoff, E., Chen, J., \& Stewart, A. J. (2008). The effect of previously viewed velocities on motion extrapolation. Vision Research, 48, 1884-1893.

McGraw, P. V., Whitaker, D., Skillen, J., \& Chung, S. T. (2002). Motion adaptation distorts perceived visual position. Current Biology, 12, 2042-2047.

McKee, S. P., Klein, S. A., \& Teller, D. Y. (1985). Statistical properties of forced-choice psychometric functions: Implications of probit analysis. Perception \& Psychophysics, 37, 286-298. doi:10.3758/ $\mathrm{BF} 03211350$

Morrone, M. C., Tosetti, M., Montanaro, D., Fiorentini, A., Cioni, G., \& Burr, D. C. (2000). A cortical area that responds specifically to optic flow, revealed by function magnetic resonance imaging. Nature Neuroscience, 3, 1322-1328. 
Oberfeld, D., Hecht, H., \& Landwehr, K. (2011). Effects of task-irrelevant texture motion on time-to-contact judgments. Attention, Perception, \& Psychophysics, 73, 581-596. doi:10.3758/s13414-010-0040-3

Pavan, A., Campana, G., Guerreschi, M., Manassi, M., \& Casco, C. (2009). Separate motion-detecting mechanisms for first- and second-order patterns revealed by rapid forms of visual motion priming and motion aftereffect. Journal of Vision, 9(11), 27.1-16. doi:10.1167/9.11.27

Pavan, A., Campana, G., Maniglia, M., \& Casco, C. (2010). The role of high-level visual areas in short-and longer-lasting forms of neural plasticity. Neuropsychologia, 48, 3069-3079.

Pavan, A., Cuturi, L. F., Maniglia, M., Casco, C., \& Campana, G. (2011). Implied motion from static photographs influences the perceived position of stationary objects. Vision Research, 51, 187-194.

Pavan, A., \& Mather, G. (2008). Distinct position assignment mechanisms revealed by cross-order motion. Vision Research, 48, 2260 2268.

Pelli, D. G. (1997). The VideoToolbox software for visual psychophysics: Transforming numbers into movies. Spatial Vision, 10, 437-442. doi:10.1163/156856897X00366

Pinkus, A., \& Pantle, A. (1997). Probing visual motion signals with a priming paradigm. Vision Research, 37, 541-552.

Schiff, W., \& Oldak, R. (1990). Accuracy of judging time to arrival: Effects of modality, trajectory, and gender. Journal of Experimental
Psychology: Human Perception and Performance, 16, 303-316. doi:10.1037/0096-1523.16.2.303

Sokolov, A., \& Pavlova, M. (2003). Timing accuracy in motion extrapolation: Reverse effects of target size and visible extent of motion at low and high speeds. Perception, 32, 699-706.

Théoret, H., Kobayashi, M., Ganis, G., Di Capua, P., \& Pascual-Leone, A. (2002). Repetitive transcranial magnetic stimulation of human area MT/V5 disrupts perception and storage of the motion aftereffect. Neuropsychologia, 40, 2280-2287.

Tresilian, J. R. (1995). Perceptual and cognitive processes in timeto-contact estimation: Analysis of prediction-motion and relative judgment tasks. Perception \& Psychophysics, 57, 231245.

Wall, M. B., Lingnau, A., Ashida, H., \& Smith, A. T. (2008). Selective visual responses to expansion and rotation in the human MT complex revealed by functional magnetic resonance imaging adaptation. European Journal of Neuroscience, 27, 2747-2757. doi:10.1111/j. 1460-9568.2008.06249.x

Whitney, D., \& Cavanagh, P. (2003). Motion adaptation shifts apparent position without the motion aftereffect. Perception \& Psychophysics, 65, 1011-1018.

Williams, Z. M., Elfar, J. C., Eskandar, E. N., Toth, L. J., \& Assad, J. A. (2003). Parietal activity and the perceived direction of ambiguous apparent motion. Nature Neuroscience, 6, 616-623. 